\title{
Nahrungsergänzungsmittel oder Arzneimittel?
}

\section{Bundesverwaltungsgericht fällt wegweisendes Urteil}

\begin{abstract}
Die Frage, ob ein Produkt den Verkehrsstatus eines Nahrungsergänzungsmittels (NEM) oder eines Arzneimittels (AM) hat bzw. haben kann, ist seit Jahrzehnten ein Dauerbrenner, der Hersteller und Konsumenten gleichermaßen verunsichert. Sicher ist bisher lediglich, dass ein Produkt nicht gleichzeitig NEM und AM sein kann, d.h. dass eine Abgrenzung unabdingbar erforderlich ist.
\end{abstract}

Im Bereich der Abgrenzung von NEM und AM hat sich im Lauf der Zeit ein kostenträchtiges Biotop von Behörden, Gremien, Sachverständi-

\section{Der bloße Verdacht} auf eine therapeutische Wirkung reicht nicht gen, Beratern, spezialisierten Juristen, etc. etabliert, ohne dass jedoch die erforderliche Sicherheit verbessert worden wäre. Es kommt hinzu, dass sich in Europa unterschiedliche nationale und europäische Bewertungen überlagern, die das regulatorische Chaos weiter verstärken. Für die Hersteller kann unter diesen Bedingungen das in den Verkehr bringen eines Produktes zu einem Glücksspiel mit hohem Kostenrisiko werden, für den Konsumenten geht jede Transparenz verloren. Auch die zahlreichen Urteile verschiedener Gerichte haben diese Unsicherheit nicht beheben können.

In dieser Situation ist nun kürzlich in einigen seit mehr als zehn Jahren (!) anhängigen Verfahren ein höchstrichterliches Urteil des Bundesverwaltungsgerichtes (BVerwG) ergangen bzw. die Revision der beklagten Bundesrepublik Deutschland abgewiesen worden (BVerwG 3 C 21.06, Urteil vom 25. Juli 2007). Mit diesem für Konsumenten wie Hersteller sehr erfreulichen Urteil wird die Rechtssicherheit in der Abgrenzung deutlich verbessert. Insbesondere wird mit diesem Urteil der beliebten behördlichen Praxis ein Riegel vorgeschoben, dass Produkte, die als NEM in den Verkehr gebracht werden, ohne weiteres als Arzneimittel eingestuft werden können, dann wegen fehlender Zulassung als nicht verkehrsfähig bewertet und so aus dem Markt gedrängt werden. Das Bundesverwaltungsgericht hat nun in seinem Urteil explizit ausgeführt, es gehe nicht an, zum Verzehr bestimmte Produkte einfach auf Verdacht den Arzneimitteln zuzurechnen.
Wenn zuverlässige wissenschaftliche Erkenntnisse über therapeutische Wirkungen oder gesundheitliche Risiken für Inhaltsstoffe von Nahrungsmitteln in einem bestimmten Dosierungsbereich fehlen, so ist es zukünftig unzulässig, diese Inhaltsstoffe auf bloßen Verdacht hin zu Arzneimitteln zu erklären und ihnen dann wegen dieser fehlenden Erkenntnisse die Verkehrsfähigkeit abzusprechen. Eine Einstufung von Produkten als Arzneimittel kann nach dem Urteil nur bei Vorliegen belastbarer, beweiskräftiger Erkenntnisse über therapeutische Wirkungen erfolgen und nicht bei Nicht-Vorliegen derartiger Erkenntnisse.

Ausgangspunkt der Kontroverse war beispielsweise ein NEM in Tablettenform, das $50 \mathrm{mg}$ aus Traubenkernen gewonnene Bioflavanole (oligomere Procyanidine) enthält, eine Stoffgruppe, die unstreitig in zahlreichen Nahrungsmitteln wie Rotwein oder Äpfeln vorkommt und zu der keine gesicherten Erkenntnisse über therapeutische Wirkungen oder gesundheitliche Risiken auch in höherer Dosierung vorliegen. Das Bundesamt für Verbraucherschutz und Lebensmittelsicherheit sah dieses Produkt als Arzneimittel an und sprach ihm die Verkehrsfähigkeit ab. Die dagegen gerichteten Klagen wurden von einem Verwaltungsgericht abgewiesen, anschließend wurde ihnen von einem Oberverwaltungsgericht stattgegeben. Das BVerwG hat nun die Revision der beklagten Bundesrepublik Deutschland zurückgewiesen. Dies bedeutet, dass eine Behörde, die in der Zukunft ein Nahrungsergänzungsmittel zu einem Arzneimittel erklärt, belastbares, beweiskräftiges Erkenntnismaterial für die therapeutische Wirkung vorlegen muss. Insofern kann das Urteil des BVerwG zukünftig auch für die Zulassung von Inhaltsstoffen von Nahrungsmitteln als Arzneimittel von Bedeutung sein, da auf diese von der Behörde als beweiskräftig akzeptierten Erkenntnisse zurückgegriffen werden kann.

Alles in allem ein sehr erfreuliches und überfälliges Urteil.

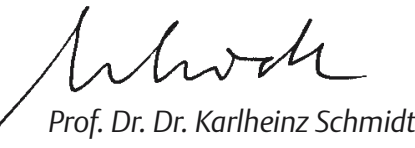

